\title{
Integral points on circles
}

\author{
A. Schinzel and M. Skałba
}

In memory of S. Srinivasan

\begin{abstract}
Sixty years ago the first named author gave an example [Sch58] of a circle passing through an arbitrary number of integral points. Now we shall prove: The number $N$ of integral points on the circle $(x-a)^{2}+(y-b)^{2}=r^{2}$ with radius $r=\frac{1}{n} \sqrt{m}$, where $m, n \in \mathbb{Z}, m, n>0$, $\operatorname{gcd}\left(m, n^{2}\right)$ squarefree and $a, b \in \mathbb{Q}$ does not exceed $r(m) / 4$, where $r(m)$ is the number of representations of $m$ as the sum of two squares, unless $n \mid 2$ and $n \cdot(a, b) \in \mathbb{Z}^{2}$; then $N \leq r(m)$.
\end{abstract}

Keywords. sums of two squares, Gaussian integers

2010 Mathematics Subject Classification. 11D25, $11 \mathrm{D} 09$.

Sixty years ago the first named author gave an example [Sch58] of a circle passing through an arbitrary number of integral points. If the center of a circle is not a rational point (i.e. not both coordinates are rational numbers) then it passes through no more than 2 rational points. In fact, the equation of the perpendicular bisector of a segment joining two rational points has rational coefficients, hence the circumcenter of a triangle with rational vertices has to be rational as well. From now on we will consider only circles

$$
(x-a)^{2}+(y-b)^{2}=r^{2},
$$

with $a, b \in \mathbb{Q}$ and we shall prove

Theorem 0.1. The number $N$ of integral points on the circle (0.1) with radius $r=\frac{1}{n} \sqrt{m}$, where $m, n \in \mathbb{Z}, m, n>0, \operatorname{gcd}\left(m, n^{2}\right)$ squarefree does not exceed $r(m) / 4$, where $r(m)$ is the number of representations of $m$ as the sum of two squares, unless $n \mid 2$ and $n \cdot(a, b) \in \mathbb{Z}^{2}$; then $N \leq r(m)$.

Lemma 0.2. Assume that $\beta, \gamma_{1}, \gamma_{2} \in \mathbb{Z}[i]$ and $c \in \mathbb{N}$ satisfy

$$
\begin{aligned}
& N\left(\gamma_{1}\right)=N\left(\gamma_{2}\right)=c^{2}, \\
& \beta \gamma_{1} \equiv \beta \gamma_{2} \quad(\bmod c),
\end{aligned}
$$

if a rational prime $t$ divides $c$ then $t \not \beta \gamma_{1}$ and $t \not \beta \gamma_{2}$.

Then $\gamma_{1} \sim \gamma_{2}$ in $\mathbb{Z}[i]$.

Proof. We assume from the beginning that $\gamma_{1} \neq \gamma_{2}$.

1. Case $\operatorname{gcd}(\beta, c) \sim 1$ : We can divide the congruence (0.3) by $\beta$ and obtain

$$
\gamma_{1}-\gamma_{2}=c \delta \quad \text { with } \delta \in \mathbb{Z}[i], \delta \neq 0 .
$$

Further

$$
N\left(\gamma_{1}\right)+N\left(\gamma_{2}\right)-\gamma_{1} \overline{\gamma_{2}}-\gamma_{2} \overline{\gamma_{1}}=c^{2} N(\delta) .
$$

If we put $\gamma_{1} \overline{\gamma_{2}}=f+g i$ with $f, g \in \mathbb{Z}$ then by equation (0.2) we obtain

$$
2 f=(2-N(\delta)) c^{2}
$$

We thank episciences.org for providing open access hosting of the electronic journal Hardy-Ramanujan Journal 
Hence

$$
f=\frac{u}{2} \cdot c^{2} \quad \text { with } u \in \mathbb{Z}, u \leq 1
$$

Because

$$
f^{2}+g^{2}=N\left(\gamma_{1} \overline{\gamma_{2}}\right)=c^{4} \quad \text { by }(0.2)
$$

one obtains

$$
g^{2}=c^{4}-f^{2}=c^{4}\left(1-\frac{u^{2}}{4}\right) .
$$

It follows $u \in\{-2,-1,0,1\}$ but $u \in\{-1,1\}$ would lead to $g \notin \mathbb{Q}$. Hence $u \in\{0,-2\}$.

If $u=0$ then $f=0, g= \pm c^{2}$ hence

$$
\gamma_{1} \overline{\gamma_{2}}= \pm c^{2} i \quad \text { what gives } \gamma_{1} c^{2}= \pm c^{2} i \gamma_{2},
$$

and finally $\gamma_{1}= \pm i \gamma_{2}$.

If $u=-2$ then $f=-c^{2}, g=0$ hence $\gamma_{1} \overline{\gamma_{2}}=-c^{2}$ and $\gamma_{1}=-\gamma_{2}$.

2. Case $N(\operatorname{gcd}(\beta, c))=d>1$ : We adopt inductive method and assume that the assertion of lemma holds for $N(\operatorname{gcd}(\beta, c))<d$. Let $\pi$ be a prime element of the ring $\mathbb{Z}[i]$ satisfying $\pi \mid \beta$ and $\pi \mid c$. By condition (0.4) (and (0.2)) $N(\pi)=p$ is a rational prime of the form $4 k+1$.

By (0.2) $\pi \mid \gamma_{1}$ or $\bar{\pi} \mid \gamma_{1}$, but the latter is excluded by (0.4), hence $\pi^{2 l} \| \gamma_{1}$ where $p^{l}|| c$. In the same way $\pi^{2 l} \| \gamma_{2}$. Rewrite the initial equality

$$
\beta \gamma_{1}-\beta \gamma_{2}=\delta c \quad \text { with } \delta \in \mathbb{Z}[i], \delta \neq 0
$$

in the form

$$
\beta \frac{\gamma_{1}}{\pi^{2 l}}-\beta \frac{\gamma_{2}}{\pi^{2 l}}=\frac{\delta \bar{\pi}^{2 l}}{p^{l}} \cdot \frac{c}{p^{l}}
$$

where all fractions are algebraic integers. Using the inductive assumption finishes the proof of lemma.

Proof of Theorem. The considered circle (0.1) is given by the equation

$$
(x-a)^{2}+(y-b)^{2}=\frac{m}{n^{2}} .
$$

Put $a=A / C, b=B / C$, where $A, B, C \in \mathbb{Z}, C>0,(A, B, C)=1$. It follows that $n \mid C$ and hence $C=n c$ with $c \in \mathbb{N}$. The number $N$ of integral points on the circle (0.5) satisfies

$$
N=\operatorname{card}\left\{(x, y) \in \mathbb{Z}^{2} \mid(C x-A)^{2}+(C y-B)^{2}=c^{2} m\right\} .
$$

Each solution $(x, y) \in \mathbb{Z}^{2}$ to the equation

$$
(C x-A)^{2}+(C y-B)^{2}=c^{2} m
$$

is encoded by the equality

$$
(C x-A)+(C y-B) i=\beta \cdot \gamma
$$

with $\beta, \gamma \in \mathbb{Z}[i]$ and $N(\beta)=m, N(\gamma)=c^{2}$.

Assume now to the contrary that the number of solutions $(x, y) \in \mathbb{Z}^{2}$ to the equation (0.6) exceeds $r(m) / 4$. It follows that there exist $\beta_{1}, \beta_{2}, \gamma_{1}, \gamma_{2} \in \mathbb{Z}[i]$ satisfying

$$
\begin{gathered}
\beta_{1} \sim \beta_{2}, N\left(\beta_{1}\right)=N\left(\beta_{2}\right)=m, N\left(\gamma_{1}\right)=N\left(\gamma_{2}\right)=c^{2}, \\
\gamma_{1} \beta_{1} \equiv \gamma_{2} \beta_{2} \quad(\bmod c) \text { and } \gamma_{1} \beta_{1} \neq \gamma_{2} \beta_{2} .
\end{gathered}
$$

Adjusting $\gamma_{1}, \gamma_{2}$ for a unit (if necessary) we may assume that there are $\beta, \gamma_{1}, \gamma_{2} \in \mathbb{Z}[i]$ satisfying

$$
N(\beta)=m, N\left(\gamma_{1}\right)=N\left(\gamma_{2}\right)=c^{2}, \beta \gamma_{1} \equiv \beta \gamma_{2} \quad(\bmod c), \gamma_{1} \neq \gamma_{2} .
$$


Now we infer by Lemma that

$$
\gamma_{2} \in\left\{-\gamma_{1}, i \gamma_{1},-i \gamma_{1}\right\}
$$

((0.4) is fulfilled by the assumption $(A, B, C)=1$.) In all above cases we get

$$
2 \beta \gamma_{1} \equiv 0 \quad(\bmod c) .
$$

For $c>2$ this contradicts the condition $(A, B, C)=1$. In case $c=2$, for any integers $A, B$ and $C \equiv 0$ $(\bmod 2)$ the conditions $(A, B, C)=1$ and

$$
(C x-A)^{2}+(C y-B)^{2}=4 m
$$

are incompatible. Concluding: $N>r(m) / 4$ is possible only for $c=1$. In case $c=1, C=n$ and by (0.6) one gets $N \leq r(m)$. It remains to deduce $n \mid 2$ from $N>r(m) / 4$. It follows from the last inequality that there exist integers $x_{1}, x_{2}, y_{1}, y_{2}$ and $k \in\{1,2,3\}$ satisfying

$$
\left(n x_{2}-A\right)+\left(n y_{2}-B\right) i=i^{k}\left[\left(n x_{1}-A\right)+\left(n y_{1}-B\right) i\right]
$$

hence

$$
\left(1-i^{k}\right)(A+B i) \equiv 0 \quad(\bmod n) .
$$

It follows $n \mid(2 A, 2 B)$ and since $(A, B, n)=1$ we infer that $n \mid 2$.

Remark. The number $1 / 4$ in our theorem is optimal and here is an example. Let $m$ be of the form $3 k+2$ and satisfying $r(m)>0$. The equality $m=x^{2}+y^{2}$ implies $x \equiv \pm 1(\bmod 3), y \equiv \pm 1(\bmod 3)$. It follows that $(x-1 / 3)^{2}+(y-1 / 3)^{2}=m / 9$ has $r(m) / 4$ integer solutions.

\section{References}

[Sch58] A. Schinzel, Sur l'existence d'un cercle passant par un nombre donne de points aux coordonnees entieres, Enseignement Math. 4 (1958), 71-72; A. Schinzel, Selecta, vol.1, 17.

\section{A. Schinzel}

Institute of Mathematics

Polish Academy of Sciences

Sniadeckich 8

00-656 Warszawa, Poland e-mail: schinzel@impan.pl

\section{Skałba}

Institute of Mathematics

University of Warsaw

Banacha 2

02-097 Warszawa, Poland e-mail: skalba@mimuw.edu.pl 